\title{
Research Article \\ Selection of an Appropriate Mechanized Mining Technical Process for Thin Coal Seam Mining
}

\author{
Chen Wang ${ }^{1}$ and Shihao Tu ${ }^{1,2}$ \\ ${ }^{1}$ School of Mines, China University of Mining and Technology, Xuzhou 221116, China \\ ${ }^{2}$ Key Laboratory of Deep Coal Resource Mining, Ministry of Education, China University of Mining and Technology, \\ Xuzhou 221116, China
}

Correspondence should be addressed to Shihao Tu; 826192989@qq.com

Received 11 July 2015; Accepted 5 October 2015

Academic Editor: Jian Guo Zhou

Copyright (C) 2015 C. Wang and S. Tu. This is an open access article distributed under the Creative Commons Attribution License, which permits unrestricted use, distribution, and reproduction in any medium, provided the original work is properly cited.

\begin{abstract}
Mechanized mining technical process (MMTP) related to the control method of the shearer is a vital process in thin coal seam mining operations. An appropriate MMTP is closely related to safety, productivity, labour intensity, and efficiency. Hence, the evaluation of alternative MMTP is an important part of the mining design. Several parameters should be considered in MMTP evaluation, so the evaluation is complex and must be compliant with a set of criteria. In this paper, two multiple criteria decisionmaking (MCDM) methods, Analytic Hierarchy Process (AHP) and Preference Ranking Organization Method for Enrichment Evaluation (PROMETHEE), were adopted for this evaluation. Then, the most appropriate MMTP for a thin coal seam working face was selected in China.
\end{abstract}

\section{Introduction}

The reserves of thin coal seam (less than $1.3 \mathrm{~m}$ in thickness) are enormous in China. Among 95 national key coal enterprises, a total of more than 750 thin coal seams exist in 445 coal mines. The recoverable reserves of thin seam are about 6.5 billion tons, accounting for $19 \%$ of the total recoverable coal reserves [1-5]. In recent years, the intensity of coal mine excavation in China has been remaining significantly high. As a result of the predatory mining principle which focuses on thick coal seams and abandons thin coal seams, the mineable reserves of thick and medium-thick coal seams were exhausted in the eastern coal mines and some aged coal mines in China. In order to balance the productivity of these coal mines and extend their service life, it was of great importance to excavate thin coal seams in many minefields, such as minefields at Huaibei, Huainan, Zibo, Yanzhou, Xuzhou, Datong, Xinwen, Hancheng, Handan, and Yulin.

Currently, the intensity of excavating thin coal seams in the above minefields is increasing year by year. However, mechanized mining of thin coal seams develops slowly due to the special mining conditions. Limited by the detrimental factors, such as high labor intensity, low degree of mechanization, low safety level, and low economic efficiency, the production of thin coal seams takes merely $10.4 \%$ of the total coal production nationwide [4] which is extremely inharmonious with respect to the recoverable reserves. Therefore, research and development of high efficient mechanized excavating techniques for thin coal seams is imminent.

Currently, mechanized mining of thin seams is mainly focused on the horizontal and slightly inclined coal seams. Relatively developed fully mechanized mining techniques include longwall mining involving coal shearer, or coal plough, auger mining, and room and pillar mining by continuous miners. The latter two techniques have been rarely used due to their low recovery rates in China [6]. Limited by factors such as poor ability of coal-rock breaking, low stability, and harsh requirements on geologic conditions, the promotion and application of coal plough in mechanized working face of thin coal seam in China is limited. However, due to high cutting efficiency, high ability of coal-rock breaking, and good adaptability, mining involving coal shearer is the primary approach of mechanized excavation of thin coal seam in China. According to incomplete statistics, among the mechanized working faces of thin coal seam in China, 
those involving coal shearer take up $85 \%$. Hence, the default mechanized mining technique of thin coal seam involved in this paper is the one involving coal shearer.

With the development of mechanizing equipment for thin coal seam, the corresponding mechanized excavating technical modes have been improved. According to the controlling method of the shearer, mechanized mining technical process (MMTP) of thin coal seam working face in China can be categorized into conventional MMTP, end-controlled MMTP [7], subdivision controlled MMTP [8,9], MMTP with memory assisted cutting $[10,11]$, and MMTP with presetting trajectory cutting [12]. These processes are applied in Zibo, Yanzhou, Huainan, Huaibei, Handan, Shuozhou, Hancheng, Shenmu, Fugu, and Dazhou coal mining area. It is well known that an appropriate MMTP is the basis for safety, productivity, and efficiency. Obviously, it is very significant to select the most appropriate MMTP. The factors to be considered in deciding MMTP include geological, economic, technical, and human factors. Therefore, MMTP selection is considered to be a decision-making process. The process can be aided by multiple criteria decision-making (MCDM) method. The general objective of MCDM is to assist the decision-maker in selecting the most suitable alternative from a number of feasible alternatives using multiple choice criteria and diverse criterion priorities [13].

\section{Findings of Existing Research}

Selecting the most appropriate MMTP is a multicriterion and multiobjective decision bound by a set of constraints. In the literature, there are many applications of decisionmaking techniques. One possible solution considered that the complexities encountered in this decision could be accomplished by the Analytic Hierarchy Process (AHP) and fuzzy PROMETHEE (Preference Ranking Organization Method for Enrichment Evaluation) method [14, 15]. Nguyen et al. presented a hybrid approach of the fuzzy ANP (Analytic Network Process) and COPRAS-G (Complex Proportional Assessment of Alternatives with Grey Relations) for fuzzy multiattribute decision-making with consideration of the interactions of the attributes [16]. To make mining method selection for the given ore bodies, Guray et al. developed a system based on 13 different expert systems and one interface agent, in which an inexperienced user can enter the system to complete his/her missing knowledge about mining method selection [17].

With respect to coal mining, Liu et al. improved the grey cluster method by assigning weights for criteria. By the cluster analysis, the technical and economic effects of three alternative mining methods including fully mechanized mining, conventional mechanized mining, and blast mining were conducted [18]. Using the comprehensive fuzzy evaluation method, Zhang et al. established a model for geological evaluation of coal mine and constructed an economicmathematical model for mining method selection for thick coal seams [19]. Based on fuzzy statistical analysis and grey relational analysis, Gao and Yang put forward the theory for comprehensive evaluation of the geological conditions of a fully mechanized working face [20]. Moreover, Monte Carlo and fuzzy AHP were also applied in selection of optimum mining method for bauxite and salt mine, respectively [21, 22]. The studies above were mainly focused on the mining method selection. However, there was very little research on the MMTP selection during mining stage, especially in thin coal seam mining working face.

The main objective of this study is to select the most appropriate MMTP for thin coal seam combining AHP and PROMETHEE method. Decision-making criteria of the study that can be effective for the selection process were defined from the literature and field observation. This paper is divided into six sections. Firstly, there is an introduction of the studied problem and a literature research. Secondly, MCDM model proposed is briefly described. Then, a group of single weights was obtained from AHP. The next section presents AHP and PROMETHEE approach for MMTP evaluation. The section before the last involves an application of the proposed approach used for a real world example. The last section of this paper concludes the study with the discussion of the results.

\section{Multiple Criteria Decision-Making Model}

Taking multiple factors into consideration, the MCDM system for MMTP selection of thin coal seam working face was established. These factors were obtained from index data of the links in a typical fully mechanized mining process. The system was divided into four layers: the Goal $(G)$ layer was MMTP selection; the Criteria $(P)$ layer was composed of economic, technical, and ergonomic factors; the Indicators $(X)$ layer was made up of eight evaluation indicators, such as equipment investment and wage; the Alternatives $(A)$ layer consisted of the four alternative MMTPs involving conventional MMTP $\left(A_{1}\right)$, end-controlled MMTP $\left(A_{2}\right)$, manual subdivision controlled MMTP $\left(A_{3}\right)$, automatic subdivision controlled MMTP $\left(A_{4}\right)$, and MMTP with presetting trajectory cutting $\left(A_{5}\right)$, as illustrated in Figure 1.

\section{MMTP Review}

In the conventional MMTP, the operators of the shearer are involved in the simultaneous controlling shearer to complete the coal cutting operation. In the end-controlled MMTP, the operators are involved in operating the shearer at both ends of the working face to complete the process of coal cutting. Manual subdivision controlled MMTP is to divide the working face reasonably according to the distance of remote control of the shearer. The shearer is operated remotely by operators in designated subdivisions in turns to complete the coal cutting process. For automatic subdivision controlled MMTP, the subdivisional location of the shearer is measured by the remote control center according to the positioning devices. According to the stored subdivisional information, remote instructions are sent out to complete the coal cutting process. During automatic subdivision control, the full length of the working face is first subdivided and the subdivisional information is stored on the control center of the shearer. The shearer only receives the remote instructions sent by the radio 


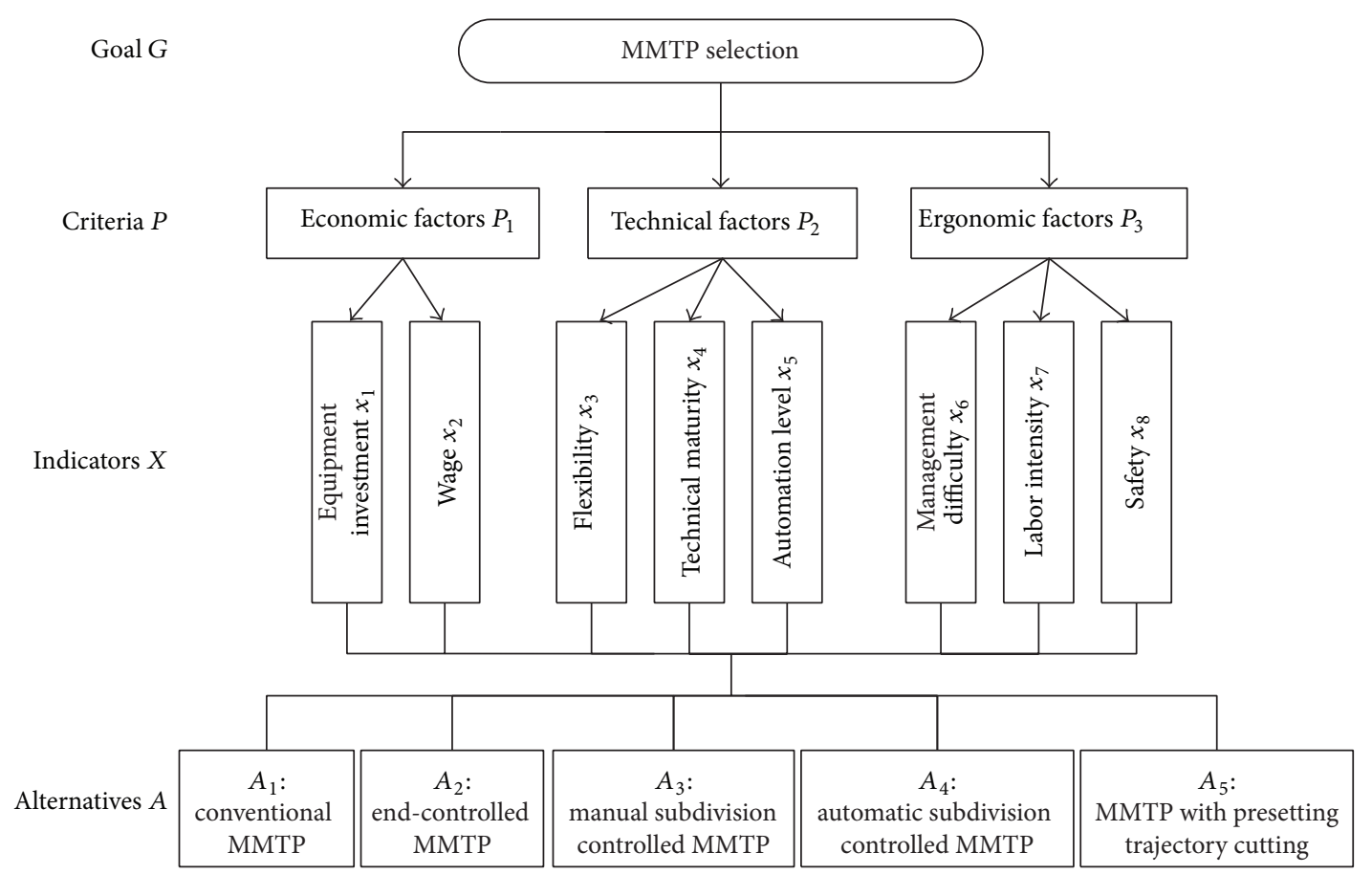

Figure 1: MCDM model for MMTP selection.

TABLE 1: Technical comparison of MMTPs.

\begin{tabular}{lll}
\hline MMTP & Advantages & Disadvantages \\
\hline Conventional & $\begin{array}{l}\text { Lower equipment investment; simple } \\
\text { technology; high maturity }\end{array}$ & $\begin{array}{l}\text { High labour intensity; poor man-machine } \\
\text { environment }\end{array}$ \\
\hline End-controlled & Liberating operators of shearer & Poor adaptability \\
\hline Manual subdivision controlled & $\begin{array}{l}\text { Lower equipment investment; reducing labour } \\
\text { intensity }\end{array}$ & $\begin{array}{l}\text { Adding several operators in site; lower safety } \\
\text { degree }\end{array}$ \\
\hline Automatic subdivision controlled & Liberating operators of shearer & $\begin{array}{l}\text { Adding one operator in site; higher automated } \\
\text { equipment investment; very poor adaptability }\end{array}$ \\
\hline Presetting trajectory cutting & $\begin{array}{l}\text { Liberating operators of shearer; high } \\
\text { adaptability }\end{array}$ & $\begin{array}{l}\text { High exploration input before mining; lower } \\
\text { maturity }\end{array}$ \\
\hline
\end{tabular}

transmitter in that subdivision. Memory assisted cutting is adopted in this MMTP. However, the location and state parameters of the shearer should be timely corrected by the operator according to the feedback from video surveillance system at the working face when the thickness of the thin seam varied or the geologic exploration is unidentified. As the latest one, MMTP with presetting trajectory cutting is operated with the operating parameters of shearer preset. Before presetting the parameters, 3D geological model consisting of thickness of seam, gas, structure, and so forth should be established. As a result, shearer can be operated in the parameters preset with unmanned intervention on site. The technical comparison among alternative MMTPs is presented in Table 1.

\section{The Analytic Hierarchy Process}

The AHP, proposed by an operational research scientist named Saaty in 1980, is a decision-making technique with qualitative and quantitative analysis. It is well adapted to complex decision situations with complicated structure of hierarchy and relative lack of necessary data. Based on the decision criteria system for MMTP selection above, the AHP was used to assign weights for each criterion. The process normally includes three steps: constructing the hierarchy judgment matrices, calculating the hierarchy relative weights, and checking the consistency of the judgments [23, 24].

5.1. Hierarchy Judgment Matrix. In this study, supposing a set of alternatives $a=\left[A_{1}, A_{2}, \ldots, A_{m}\right]$ including $m$ alternatives and a set of evaluation indicators $X=\left[X_{1}, X_{2}, \ldots, X_{n}\right]$ including $n$ indicators, the elements on each level were pairwise compared. Based on the pairwise comparisons, judgments were made about their importance by reference to the comparison criteria table (Table 2) [23]. Then, according to the results from pairwise comparisons, hierarchy judgment matrices $R$ composed of $R(G-P)$ and $R(P-X)$ were created, as shown in Table 3. 
TABLE 2: Scale for pairwise comparisons.

\begin{tabular}{|c|c|c|}
\hline Relative intensity & Definition & Explanation \\
\hline 1 & Of equal value & Two requirements are of equal value \\
\hline 3 & Slightly higher value & Experience slightly favors one requirement over another \\
\hline 5 & Essential or strong value & Experience strongly favors one requirement over another \\
\hline 7 & Very strong value & $\begin{array}{l}\text { A requirement is strongly favored and its dominance is demonstrated } \\
\text { in practice }\end{array}$ \\
\hline 9 & Extreme value & $\begin{array}{l}\text { The evidence favoring one over another is of the highest possible } \\
\text { order of affirmation }\end{array}$ \\
\hline $2,4,6$, and 8 & $\begin{array}{l}\text { Intermediate values } \\
\text { between two adjacent } \\
\text { judgments }\end{array}$ & When compromise is needed \\
\hline
\end{tabular}

TABLE 3: AHP judgement matrix.

\begin{tabular}{lcccccccccccccc}
\hline$R(G-P)$ & $P_{1}$ & $P_{2}$ & $P_{3}$ & $R\left(P_{1}-X\right)$ & $x_{1}$ & $x_{2}$ & $R\left(P_{2}-X\right)$ & $x_{3}$ & $x_{4}$ & $x_{5}$ & $R\left(P_{3}-X\right)$ & $x_{6}$ & $x_{7}$ & $x_{8}$ \\
\hline$P_{1}$ & 1 & $1 / 2$ & 1 & $x_{1}$ & 1 & 1 & $x_{3}$ & 1 & 3 & 2 & $x_{6}$ & 1 & $1 / 4$ & $1 / 3$ \\
$P_{2}$ & 2 & 1 & 1 & $x_{2}$ & 1 & 1 & $x_{4}$ & $1 / 3$ & 1 & $1 / 3$ & $x_{7}$ & 4 & 1 & 2 \\
$P_{3}$ & 1 & 1 & 1 & & & & $x_{5}$ & $1 / 2$ & 3 & 1 & $x_{8}$ & 3 & $1 / 2$ & 1 \\
\hline
\end{tabular}

5.2. Hierarchy Relative Weight. In general, to meet the requirements of the precision in calculation of the relative weight by using the AHP, it is enough to conduct an approximate calculation using the square root method as follows.

Calculating the geometric means of the elements on each row of the judgment matrices,

$$
\bar{\omega}_{i}=\sqrt[n]{\prod_{j=1}^{n} a_{i j}}, \quad i=1,2, \ldots, n,
$$

where $a_{i j}$ represents the elements of the judgment matrix, so that $\bar{\omega}=\left(\bar{\omega}_{1}, \bar{\omega}_{2}, \ldots, \bar{\omega}_{n}\right)^{\mathrm{T}}$. Then, $\bar{\omega}_{i}$ is normalized as follows:

$$
\omega_{i}=\frac{\bar{\omega}_{i}}{\sum_{i=1}^{n} \bar{\omega}_{i}}, \quad i=1,2, \ldots, n
$$

Then, the weight vector $\omega=\left(\omega_{1}, \omega_{2}, \ldots, \omega_{n}\right)^{\mathrm{T}}$ was obtained. According to the definition of combination weight in the AHP, the relative weight vector of the Indicators with respect to the Goal was obtained and can be illustrated as follows:

$\omega$

$$
=(0.130,0.130,0.218,0.058,0.137,0.040,0.183,0.105)^{\mathrm{T}} .
$$

Thus, adaptability and labor intensity are the most important criteria for MMTP selection based on the opinion survey.

5.3. Checking the Consistency. The consistency of the judgment matrices must be checked to measure its credibility. The consistency criterion used is $C_{R}$ :

$$
C_{R}=\frac{1}{R_{I}} \cdot \frac{\lambda_{\max }-n}{n-1}
$$

TABle 4: Consistency indices of randomly generated reciprocal matrices.

\begin{tabular}{lccccccc}
\hline Order of matrix & 1 & 2 & 3 & 4 & 5 & 6 & 7 \\
\hline$R_{I}$ value & 0.00 & 0.00 & 0.58 & 0.90 & 1.12 & 1.24 & 1.32 \\
\hline
\end{tabular}

TABLE 5: Consistency test results of AHP judgment matrix.

\begin{tabular}{lccc}
\hline Judgment matrix & $n$ & $\lambda_{\max }$ & $C_{R}$ \\
\hline$G-P$ & 3 & 3.0536 & 0.0462 \\
$P_{1}-X$ & 2 & 2 & 0 \\
$P_{2}-X$ & 3 & 3.0536 & 0.0462 \\
$P_{3}-X$ & 3 & 3.0183 & 0.0158 \\
\hline
\end{tabular}

where $R_{I}$ is the correction value (Table 4 ), depending on the dimensions of the matrices; $\lambda_{\max }$ is the maximum eigenvalue of the matrices:

$$
\lambda_{\max }=\sum_{i=1}^{n} \frac{(R \bar{\omega})_{i}}{n \bar{\omega}_{i}}
$$

where $R$ is the judgment matrix and $(R \bar{\omega})_{i}$ represents the $i$ th element of the vector $R \bar{\omega}$. If $C_{R} \leqslant 0.1$, the consistency of the matrix is considered to be acceptable and the weight vectors derived from it are credible. Otherwise, the judgment matrices need to be reconstructed until their consistency can meet the demands.

By the formula above, the consistency checking results of the judgment matrices were obtained, as shown in Table 5. The results showed that the consistency of judgement matrices can meet the requirements.

\section{Fuzzy PROMETHEE Method}

Claimed by Belgian Professor Brans in 1984, PROMETHEE is a method used in MCDM problems to rank the alternatives, 
which takes advantage of preference function, criterion value, and criterion weight given by decision-makers to determine the scheme with optimal order [25].

6.1. PROMETHEE Method. Suppose scheme $A=\left(a_{1}, a_{2}\right.$, $\left.\ldots, a_{m}\right)$. Comprehensive ordering is operated under the condition of criteria set $c=\left(f_{1}, f_{2}, \ldots, f_{n}\right)$ and weight vector $W_{k}=\left(w_{1}, w_{2}, \ldots, w_{n}\right)$. As a result, the optimal order intensity $G_{k}\left(d_{i j}\right)$ of $a_{i}$ and $a_{j}$ under the condition of criteria $c$ is the preference function value of criterion value difference and can be expressed using the function as follows:

$$
G_{k}\left(d_{i j}\right)=P_{k}\left(a_{i}, a_{j}\right) \in[0,1] .
$$

The optimal order intensity of $a_{i}$ goes from $G_{k}(d)=0$ (no difference) to $G_{k}(d)=1$ (strict priority) relative to that of $a_{j}$. The multiattribute preference optimal sequence index can be expressed as follows:

$$
H\left(a_{i}, a_{j}\right)=\sum_{k=1}^{n} W_{k} P_{k}\left(a_{i}, a_{j}\right) .
$$

In PROMETHEE I, the positive direction and the negative direction of preference priority rating of $a_{i}$ can be, respectively, shown as follows:

$$
\begin{aligned}
& \Phi^{+}\left(a_{i}\right)=\sum_{j=1}^{m} H\left(a_{i}, a_{j}\right), \\
& \Phi^{-}\left(a_{i}\right)=\sum_{j=1}^{m} H\left(a_{j}, a_{i}\right),
\end{aligned}
$$

where $\Phi^{+}\left(a_{i}\right)$ is the degree by which $a_{i}$ is superior to $a_{j}$ and $\Phi^{-}\left(a_{i}\right)$ is the degree by which $a_{i}$ is inferior to $a_{j}$. The scheme is superior to the higher positive direction or the lower negative direction. Complete sorting of the scheme set can be obtained according to PROMETHEE II. The optimal relationship of different schemes depends on comprehensive optimal level value, which can be expressed as follows:

$$
\Phi\left(a_{i}\right)=\Phi^{+}\left(a_{i}\right)-\Phi^{-}\left(a_{i}\right) .
$$

6.2. Preference Function. Brans and other professors have offered six kinds of preference functions $[15,21,25,26]$. In this research, linear preference function with no difference interval was adopted. The accuracy of the results can meet the needs of solving this problem. Linear preference function can be expressed as follows:

$$
P_{k}\left(a_{i}, a_{j}\right)= \begin{cases}0 & d \leq 0 \\ \frac{d_{i j}}{p} & 0<d \leq p \\ 1 & d>p,\end{cases}
$$

where $d_{i j}=x_{i r}-x_{j r}(i, j=1,2, \ldots, m ; r=1,2, \ldots, n), x_{i j}$ is the element of the judgment matrix, and $p_{r}$ is the strict preference threshold value of the linear preference function to $r$ th criterion, which can be expressed as $p_{r}=\max \left(x_{i r}\right)-$ $\min \left(x_{i r}\right)$ [27].

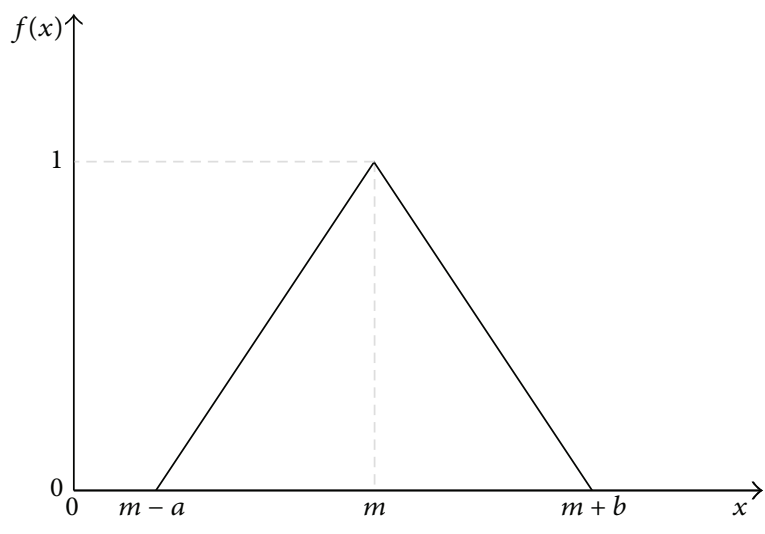

Figure 2: Triangular fuzzy numbers.

6.3. Judgment Matrix. In this study, supposing a set of alternatives $a=\left[A_{1}, A_{2}, \ldots, A_{m}\right]$ including $m$ alternatives and a set of evaluation indicators $X=\left[x_{1}, x_{2}, \ldots, x_{n}\right]$ including $n$ indicators, $x_{i j}(i=1,2, \ldots, m ; j=1,2, \ldots, n)$ represents the $j$ th indicator of the $i$ th alternative $A_{i}$. Thus, the judgment matrix can be expressed as

$$
R=\left(X_{i j}\right)_{m \times n}=\left[\begin{array}{cccc}
x_{11} & x_{12} & \cdots & x_{1 n} \\
x_{21} & x_{22} & \cdots & x_{2 n} \\
\vdots & \vdots & \vdots & \vdots \\
x_{m 1} & x_{m 2} & \cdots & x_{m n}
\end{array}\right] \text {. }
$$

Obviously, some evaluation indicators could not be quantified accurately in this paper, such as the adaptability $\left(x_{3}\right)$, technical maturity $\left(x_{4}\right)$, automation level $\left(x_{5}\right)$, management difficulty $\left(x_{6}\right)$, and labor intensity $\left(x_{7}\right)$. Therefore, the fuzzy set theory was introduced and applied to quantify these indicators.

6.3.1. Fuzzy Set Theory. $f(x)$ is about the membership function of fuzzy number $x$, in which $x=(m, a, b)_{L R}$; that is to say, $m-a<x<m+b$ and $f(x) \in[0,1]$. Within the scope of $(m-a, m)$ and $(m, m+b), f(x)$ is linear monotone increasing function and monotone decreasing function, respectively [28], as shown in Figure 2.

In this paper, the decision-makers had 6 choices [29], namely, $\mathrm{VG}(1,0.2,0)_{L R}, \mathrm{G}(0.8,0.2,0.2)_{L R}, \mathrm{M}(0.6,0.2,0.2)_{L R}$, $\mathrm{W}(0.4,0.2,0.2)_{L R}, \mathrm{~B}(0.2,0.2,0.2)_{L R}$, and $\mathrm{VB}(0,0,0.2)_{L R}$.

In the evaluation, equipment investment $\left(x_{1}\right)$, wage $\left(x_{2}\right)$, and safety degree $\left(x_{8}\right)$ are predictable cost indicators. On purpose of unification and comprehensive ordering, the other indicators expressed as fuzzy numbers in the judgment matrix should be defuzzed into cost index. Table 6 showed the fuzzy values of the indicators obtained in the fuzzy evaluation.

6.3.2. Defuzzy. Eventually, in the initial judgment matrix, we could foresee from the introduced methodology that the results obtained will be fuzzy numbers, and, according to a 
TABLE 6: Fuzzy expressions of evaluation index.

\begin{tabular}{|c|c|c|c|c|c|c|c|}
\hline Number & Attribute value & Flexibility & Maturity & Automation & Management difficulty & Labour intensity & Yager index \\
\hline VB & $(1,0.2,0)_{L R}$ & Very inflexible & Very immature & Very low & Very hard & Very high & 0.933 \\
\hline B & $(0.8,0.2,0.2)_{L R}$ & Inflexible & Immature & Low & Hard & High & 0.800 \\
\hline W & $(0.6,0.2,0.2)_{L R}$ & General & General & General & General & General & 0.600 \\
\hline M & $(0.4,0.2,0.2)_{L R}$ & Medium & Medium & Medium & Medium & Medium & 0.400 \\
\hline G & $(0.2,0.2,0.2)_{L R}$ & Flexible & Mature & High & Easy & Low & 0.200 \\
\hline VG & $(0,0,0.2)_{L R}$ & Very flexible & Very mature & Very high & Very easy & Very low & 0.067 \\
\hline
\end{tabular}

conclusion in our selection problem, these results of fuzzy numbers have to be ranked with respect to the principles of PROMETHEE method, and this means that fuzzy numbers have to be compared. In order to compare the fuzzy numbers, Goumas and Lygerou (2000) proposed to use Yager index $[30,31]$, and we used this index to defuzz the fuzzy numbers and compare them in this study. According to this method, defuzzy form of a given fuzzy number can be calculated as follows:

$$
f(x)=F(m, a, b)=\frac{(3 m-a+b)}{3} .
$$

6.3.3. Primary Judgment Matrix. In the MMTP evaluation process, the evaluation indicators can be quantified through field research, consultation with experts, and fuzzy evaluation (Table 6). Equipment considered in the evaluation is composed of video monitoring system of shearer, geological exploration input related to MMTP, and other automated equipment. With respect to wage $\left(x_{2}\right)$ indicator, only operators of shearer are considered, including remote control personnel and workers on site. The wage can be illustrated as follows:

$$
x_{2}=w\left(d_{i}+e_{i}\right)= \begin{cases}5000(2+0) & A=A_{1} \\ 5000(0+2) & A=A_{2} \\ 5000\left(\left\{\frac{L}{l}\right\}+0\right) & A=A_{3} \\ 5000(1+1) & A=A_{4} \\ 5000(0+1) & A=A_{5},\end{cases}
$$

where $w$ is the average wage per month (5000 yuan $\mathrm{RMB} /$ month) and $d_{i}$ and $e_{i}$ are the number of workers on site and remote control personnel, respectively. $\{L / l\}$, as a function of $e$, is the smallest integer greater than or equal to $L / l, L$ is the length of thin coal seam working face $(30 \sim 300 \mathrm{~m})$, and $l$ is the remote control distance of shearer on site $(20 \mathrm{~m})$.

Safety degree $\left(x_{8}\right)$ is a function of the number of workers on site $\left(d_{i}\right)$, which can be expressed as follows:

$$
x_{8}=1-(1-\alpha)^{d_{i}},
$$

where $\alpha$ is the safety accident rate per operator of shearer in thin coal seam panel. From the above, the values of all indicators can be illustrated in Table 7 .

Thus, the judgment matrix can be expressed as follows:

$$
R=\left(X_{i j}\right)_{m \times n}=\left[\begin{array}{cccccccc}
0 & 10000 & 0.067 & 0.067 & 0.933 & 0.6 & 0.933 & 1-(1-\alpha)^{2} \\
0 & 10000 & 0.933 & 0.6 & 0.933 & 0.4 & 0.2 & 0 \\
0 & 5000 e & 0.2 & 0.2 & 0.933 & 0.8 & 0.4 & 1-(1-\alpha)^{e} \\
1.5 & 5000 & 0.8 & 0.6 & 0.2 & 0.4 & 0.2 & \alpha \\
2 & 5000 & 0.2 & 0.8 & 0.067 & 0.4 & 0.067 & 0
\end{array}\right] .
$$

\section{Results and Discussions}

At the beginning of the evaluation, all the indicators have been transformed into cost indexes. Hence, the priorities of the alternatives decrease with the values of the final evaluation obtained from AHP or PROMETHEE.

7.1. Results from AHP. The performance graph (Figure 3) depicts the value of the evaluation obtained from AHP.
With respect to the economical main criterion, the value of the evaluation $(E(e))$ is closely related to the coefficient about the length of working face $(e)$. The priorities of the alternatives were presented in Table 8. If the technical main criterion is considered, alternative $A_{5}$ is preferable to other alternatives, and the other alternatives are ranked in the order of $A_{1}, A_{3}, A_{4}$, and $A_{2}$. In the same way, if the ergonomic main criterion is considered, alternative $A_{5}$ is also selected to be the best alternative MMTP, and the other 
TABLE 7: Values of indicators.

\begin{tabular}{|c|c|c|c|c|c|}
\hline \multirow{2}{*}{ Indicators } & \multicolumn{5}{|c|}{ Alternatives } \\
\hline & $A_{1}$ & $A_{2}$ & $A_{3}$ & $A_{4}$ & $A_{5}$ \\
\hline$x_{1}($ million yuan $\mathrm{RMB})$ & 0 & 0 & 0 & 1.5 & 2.0 \\
\hline$x_{2}($ yuan $\mathrm{RMB})$ & 10000 & 10000 & $5000 e$ & 5000 & 5000 \\
\hline$x_{3}$ & VG/0.067 & $\mathrm{VB} / 0.933$ & $\mathrm{G} / 0.2$ & $\mathrm{~B} / 0.8$ & $\mathrm{G} / 0.2$ \\
\hline$x_{4}$ & VG/0.067 & W/0.6 & $\mathrm{G} / 0.2$ & $\mathrm{~W} / 0.6$ & $\mathrm{~B} / 0.8$ \\
\hline$x_{5}$ & $\mathrm{VB} / 0.933$ & $\mathrm{VB} / 0.933$ & $\mathrm{VB} / 0.933$ & G/0.2 & VG/0.067 \\
\hline$x_{6}$ & $\mathrm{~W} / 0.6$ & $\mathrm{M} / 0.4$ & $\mathrm{~B} / 0.8$ & $\mathrm{M} / 0.4$ & $\mathrm{M} / 0.4$ \\
\hline$x_{7}$ & $\mathrm{VB} / 0.933$ & $\mathrm{G} / 0.2$ & $\mathrm{M} / 0.4$ & $\mathrm{G} / 0.2$ & VG/0.067 \\
\hline$x_{8}$ & $1-(1-\alpha)^{2}$ & 0 & $1-(1-\alpha)^{e}$ & $\alpha$ & 0 \\
\hline
\end{tabular}

Notes: all indicators are cost indexes.

TABLE 8: Priorities of the alternatives considering economical main criterion.

\begin{tabular}{lcrrrrr}
\hline$e$ & $2(L=20 \sim 40 \mathrm{~m})$ & $3(L=40 \sim 60 \mathrm{~m})$ & $4(L=60 \sim 80 \mathrm{~m})$ & $\begin{array}{c}5 \sim 6 \\
(L=80 \sim 120 \mathrm{~m})\end{array}$ & $\begin{array}{c}7 \sim 10 \\
(L=120 \sim 200 \mathrm{~m})\end{array}$ & $(L=200 \sim 300 \mathrm{~m})$ \\
\hline Priorities & $A_{3}, A_{4}, A_{2} / A_{1}, A_{5}$ & $A_{3}, A_{2} / A_{1}, A_{4}, A_{5}$ & $A_{3} / A_{2} / A_{1}, A_{4}, A_{5}$ & $A_{2} / A_{1}, A_{3}, A_{4}, A_{5}$ & $A_{2} / A_{1}, A_{4}, A_{3}, A_{5}$ & $A_{2} / A_{1}, A_{4}, A_{5}, A_{3}$ \\
\hline
\end{tabular}

Notes: $A_{i} / A_{j}$ shows that the priority of alternative $A_{i}$ is the same as the one of $A_{j}$.

TABLE 9: Priorities of the alternatives considering all criteria.

\begin{tabular}{lcc}
\hline$e$ & 2 & $4 \sim 15$ \\
\hline Priorities & $A_{5}, A_{2} / A_{1}, A_{3}, A_{4}$ & $A_{5}, A_{2} / A_{1}, A_{4}, A_{3}$ \\
\hline
\end{tabular}

alternatives are ranked in the order of $A_{2}, A_{4}, A_{1}$, and $A_{3}$.

The overall weights of the alternative MMTPs were obtained by multiplying the priority of each main criterion by the priority of each alternative. As illustrated in Figure 3, the overall ranking of the alternatives can be calculated. With respect to all criteria, the value of the evaluation $(O(e))$ is closely related to the coefficient about the length of working face $(e)$.

The priorities of the alternatives considering all criteria were presented in Table 9.

7.2. Results from PROMETHEE. After the value of $H\left(A_{i}, A_{j}\right)$ is determined, alternative MMTPs are evaluated, and positive flow $\left(\Phi^{+}\left(a_{i}\right)\right)$, negative flow $\left(\Phi^{-}\left(a_{i}\right)\right)$, and net flow $\left(\Phi\left(a_{i}\right)\right)$ values are calculated. The obtained flow values are presented in Table 10. Then, the ranking results are calculated by PROMETHEE I and PROMETHEE II with the usage of positive flow and net flow values in this table. The accurate ranking of alternatives is presented in Figure 4. Table 11 shows the ranking results in detail.

Alternatives are ranked from the best to the worst one by using the net flow $\left(\Phi\left(a_{i}\right)\right)$ in PROMETHEE II complete ranking. Here, according to the compartments based on the net flows, the alternative with the higher net flow is assumed to be inferior to the others, and the rest of the alternatives are ranked due to their net flow values likewise. Since PROMETHEE I does not provide a complete ranking, resulting ranking called partial ranking cannot be compared with the ranking provided by PROMETHEE II. In some ranking problems, PROMETHEE I can give a complete ranking depending on the evaluation matrix values and this ranking cannot be different from the one achieved with PROMETHEE II. As illustrated in Figure 4, the negative flow and net flow values are closely related to the coefficient about length of working face [32].

As illustrated in Table 11, partial ranking of alternative is closely related to the coefficient about the length of working face $(e)$. There are 4 kinds of possibilities in the partial ranking. Then, complete ranking of alternatives is a must to the final ranking results. Alternative $5\left(A_{5}\right)$ is selected to be the best alternative MMTP according to the complete ranking results, and the other alternatives are ranked in the order of $A_{4}, A_{3}, A_{2}$, and $A_{1}$.

7.3. Discussions. In the coal mining engineering, only one MMTP usually cannot meet the demand of safety, productivity, and efficiency. The specific MMTP should be adopted in the given mining stage. In this paper, several kinds of MMTPs applied successfully in China were evaluated by AHP and PROMETHEE, respectively. From the above ranking results, the best alternative MMTP selected by PROMETHEE is clearly consistent with the one by AHP. The most appropriate alternative MMTP for thin coal seam mining, MMTP with presetting trajectory cutting, is selected.

Before presetting trajectory cutting, with the usage of detection techniques, 3D geological model of thin coal seam working face which can meet mining engineering requirements should be established, including thickness of seam, gas, and structure. Then, the operating parameters of shearer can be preset in some degree according to the 3D geological model. During the normal mining, shearer in thin coal seam working face can be operated in the parameters preset with unmanned intervention on site. In this way, the operators of the shearer can be liberated utterly. MMTP with presetting 
TABle 10: Fuzzy PROMETHEE flows.

\begin{tabular}{|c|c|c|c|c|c|}
\hline \multirow{2}{*}{ Flow values } & \multicolumn{5}{|c|}{$A_{i}$} \\
\hline & $A_{1}$ & $A_{2}$ & $A_{3}$ & $A_{4}$ & $A_{5}$ \\
\hline$\Phi^{+}\left(a_{i}\right)$ & $\begin{array}{c}1.375+0.26 /(e-1)+ \\
0.105\left[5 \alpha-3 \alpha^{2}\right] /[1- \\
\left.(1-\alpha)^{e}\right]\end{array}$ & $0.975+0.26 /(e-1)$ & $\begin{array}{c}1.531-0.26 /(e-1)+ \\
0.105\left(\alpha^{2}-3 \alpha\right) /[1- \\
\left.(1-\alpha)^{e}\right]\end{array}$ & $\begin{array}{c}0.901+0.21 \alpha /[1- \\
\left.(1-\alpha)^{e}\right]\end{array}$ & 0.592 \\
\hline$\Phi^{-}\left(a_{i}\right)$ & $\begin{array}{c}1.104-0.13 /(e-1)+ \\
0.105\left(\alpha^{2}-2 \alpha\right) /[1- \\
\left.(1-\alpha)^{e}\right]\end{array}$ & $\begin{array}{c}0.784-0.13 /(e-1)+ \\
0.105\left(3 \alpha-2 \alpha^{2}\right) /[1- \\
\left.(1-\alpha)^{e}\right]\end{array}$ & 1.194 & $\begin{array}{l}0.921+0.26 /(e-1)- \\
0.105 \alpha^{2} /\left[1-(1-\alpha)^{e}\right]\end{array}$ & $\begin{array}{c}1.371+0.26 /(e-1)+ \\
0.105\left(3 \alpha-\alpha^{2}\right) /[1- \\
\left.(1-\alpha)^{e}\right]\end{array}$ \\
\hline$\Phi\left(a_{i}\right)$ & $\begin{array}{c}0.271+0.39 /(e-1)+ \\
0.105\left[7 \alpha-4 \alpha^{2}\right] /[1- \\
\left.(1-\alpha)^{e}\right]\end{array}$ & $\begin{array}{c}0.191+0.39 /(e-1)- \\
0.105\left(3 \alpha-2 \alpha^{2}\right) /[1- \\
\left.(1-\alpha)^{e}\right]\end{array}$ & $\begin{array}{c}0.337-0.26 /(e-1)+ \\
0.105\left(\alpha^{2}-3 \alpha\right) /[1- \\
\left.(1-\alpha)^{e}\right]\end{array}$ & $\begin{array}{c}-0.02-0.26 /(e-1)+ \\
0.105\left(2 \alpha+\alpha^{2}\right) /[1- \\
\left.(1-\alpha)^{e}\right]\end{array}$ & $\begin{array}{c}-0.799-0.26 /(e- \\
1)-0.105(3 \alpha- \\
\left.\alpha^{2}\right) /\left[1-(1-\alpha)^{e}\right]\end{array}$ \\
\hline
\end{tabular}

TABLE 11: The resulting ranking.

\begin{tabular}{lcccc}
\hline$e$ & $2(L=20 \sim 40 \mathrm{~m})$ & $3 \sim 9(L=40 \sim 180 \mathrm{~m})$ & $10 \sim 12(L=180 \sim 240 \mathrm{~m})$ & $13 \sim 15(L=240 \sim 300 \mathrm{~m})$ \\
\hline Partial ranking & $A_{5}, A_{4}, A_{3}, A_{2}, A_{1}$ & $A_{5}, A_{4}, A_{2}, A_{3}, A_{1}$ & $A_{5}, A_{4}, A_{2}, A_{3} / A_{1}$ & $A_{5}, A_{4}, A_{2}, A_{1}, A_{3}$ \\
\hline Complete ranking & & $A_{5}, A_{4}, A_{3}, A_{2}, A_{1}$ & \\
\hline
\end{tabular}

Notes: $A_{i} / A_{j}$ shows that the priority of alternative $A_{i}$ is the same as the one of $A_{j}$.

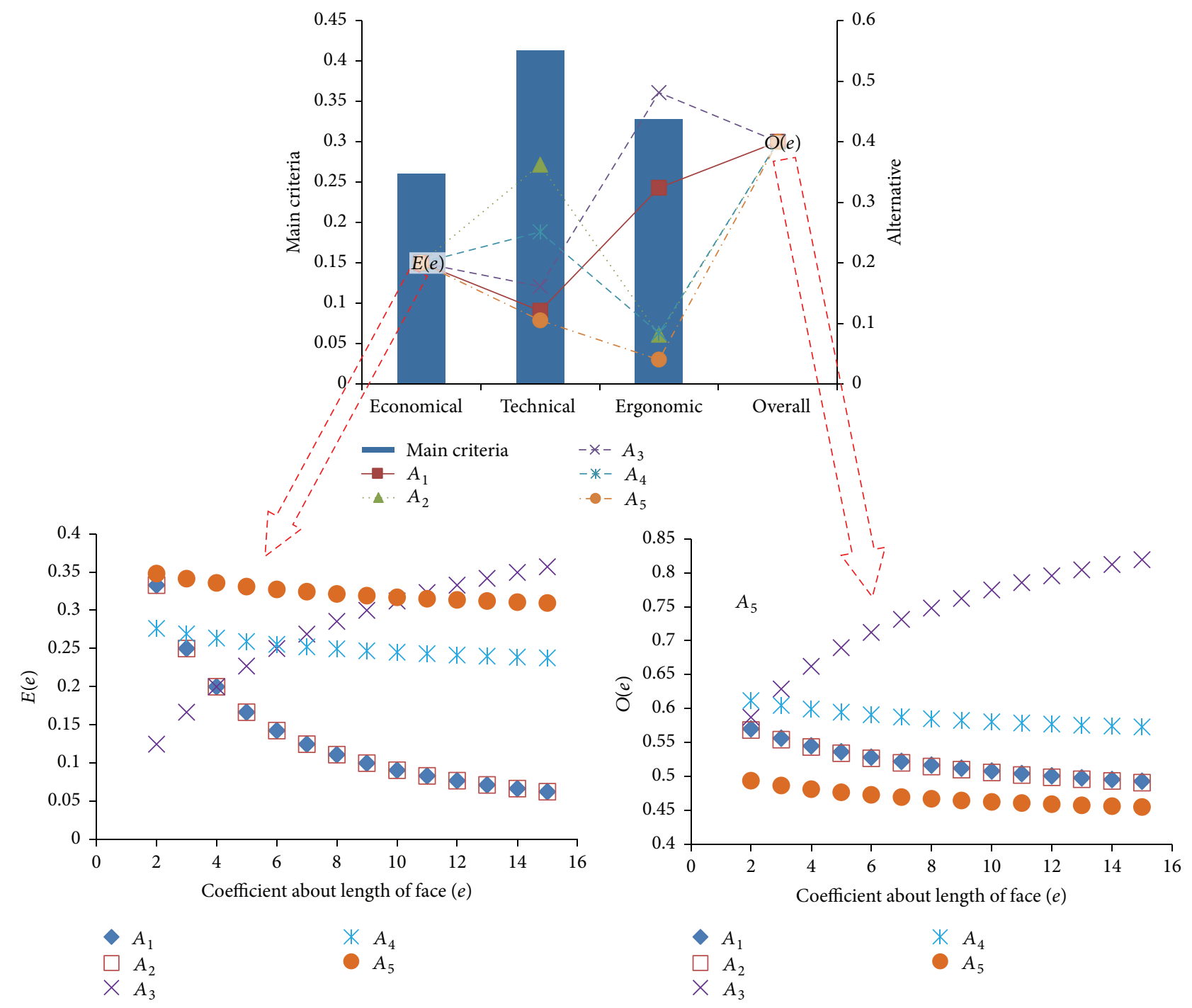

FIGURE 3: Final priorities of the five MMTPs from AHP. 

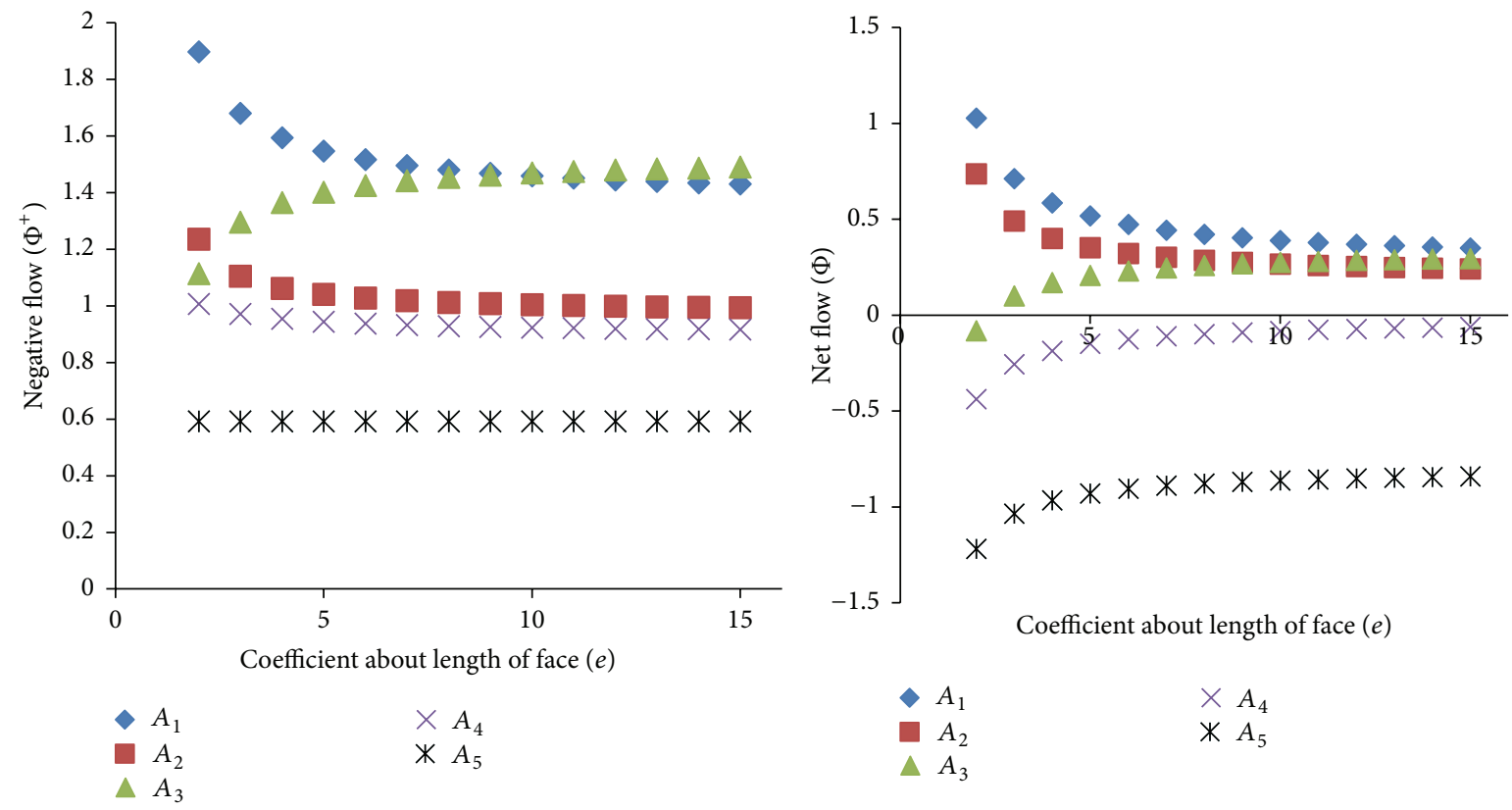

FIGURE 4: PROMETHEE ranking.

trajectory cutting will have potentially broad application in the fields of thin coal seam mining.

In general, with the increasing of precision of geologic exploration of thin coal seam and key assisting intelligent techniques, MMTP should transit from other MMTPs towards MMTP with presetting trajectory cutting, to achieve the intelligent and unmanned excavation of thin coal seam.

\section{Case Study}

Panel 43101 is a thin coal seam working face at Liangshuijing coal mine of Shaanxi Huisen Coal Industry Development Co., Ltd., in China. The panel mainly focuses on mining $\# 4^{-3}$ coal seam. Fully mechanized overall height mining is adopted in this face. The thickness of $\# 4^{-3}$ coal seam is from $1.05 \mathrm{~m}$ to $1.4 \mathrm{~m}$, and the average is $1.14 \mathrm{~m}$. The inclination is from $0^{\circ}$ to $1^{\circ}$. According to statistics, the safety accident rate per operator of shearer $(\alpha)$ in this panel is 0.01 . The length of this panel $(L)$ is $160 \mathrm{~m}$.

Based on the results from AHP and PROMETHEE in Section 7, the most appropriate MMTP, that is, MMTP with presetting trajectory cutting, for panel 43101 was selected.

In the acceptance conference of the Research on the Mining Techniques and Equipment for Thin Seamsin Xian, the most appropriate MMTP was recognized unanimously by the participating experts. Moreover, MMTP with presetting trajectory cutting is successfully applied in the thin coal seam mining for panel 43101.

\section{Conclusions}

This paper has demonstrated the application of the AHP and PROMETHEE methods in evaluating MMTPs for thin coal seam mining in China. Unlike the conventional approach which is an empirical method for the selection of MMTPs, the AHP and PROMETHEE methods make it possible to evaluate the alternative MMTPs in a more scientific manner which preserves integrity and objectivity. The two methods are flexible, transparent, easy to comprehend, and easy to apply by decision-makers.

In the established decision-making model, five alternatives were evaluated with regard to three main criteria and their subcriteria. The evaluations by the two methods revealed that the most appropriate MMTP for thin coal seam mining is MMTP with presetting trajectory cutting $\left(A_{5}\right)$. The weight calculated from AHP revealed that the technical main criterion carried the highest weight of relative importance in the evaluation process; this was a result of the fact that decision-makers pay more attention to technical criterion than the other criteria.

MMTP is a crucial task in mining operations. It is closely related to safety, productivity, and efficiency. The rational evaluation of MMTPs for thin coal seam mining requires the consideration of numerous criteria, including economical, technical, and ergonomic factors. The problem is based on the comparisons of alternative MMTPs according to the identified criteria. Hence, decision-making methods to solve MCDM problem are considered to be used in the paper. For this purpose, AHP and PROMETHEE methods work together to solve this problem, and the most appropriate MMTP was selected.

\section{Conflict of Interests}

The authors declare that there is no conflict of interests regarding the publication of this paper. 


\section{Acknowledgments}

Financial support for this work was provided by the Chinese National 863 High Technology Plan (no. 2012AA062101) and a project funded by the Priority Academic Program Development of Jiangsu Higher Education Institutions and the Fundamental Research Funds for the Central Universities (no. 2014XT01). The authors gratefully acknowledge the financial support from the organizations mentioned above.

\section{References}

[1] J. He, L.-M. Dou, and C.-P. Lu, "Characteristic and prevention research on rock burst of thin coal seam," Journal of the China Coal Society, vol. 37, no. 7, pp. 1094-1098, 2012.

[2] J.-Q. Jiang, T.-Z. Qu, J. Dai, H. Li, and Z.-C. Tian, "Numerical test of spalling of iron sulfide concretions in thin seam," Journal of the China Coal Society, vol. 34, no. 4, pp. 472-477, 2009.

[3] C. S. Han, "Technology of fully mechanized thin seam mining in Jiangjiawan mine of Datong minging area," Coal Science and Technology, vol. 41, supplement, pp. 11-12, 2013.

[4] K. W. Wang and Y. Q. Luo, "Efficient mining techniques in thin coal seam with complex structure and extra-long face," Coal Mine Modernization, no. 3, pp. 17-19, 2013.

[5] G. J. Sheng, Q. S. Sun, and H. L. Song, "The innovational mining technology of fully mechanized mining on thin coal seam," Journal of China Coal Society, vol. 32, no. 3, pp. 230-234, 2007.

[6] D. B. Mao, H. Lan, and G. Xu, "Thin coal-seam mechanized mining status and new development of China," Coal Mining Technology, vol. 16, no. 3, pp. 11-14, 76, 2011.

[7] L. Y. Li and F. T. Wang, "Equipment matching and application of fully mechanized mining face in thin seam with iron sulfide tuberculosis," Shandong Coal Science and Technology, no. 2, pp. 98-101, 2010.

[8] Z. K. Guo, "Automatic matching equipment and technology of fully coal mining face in thin seam," Coal Science and Technology, vol. 41, no. 4, pp. 24-27, 2013.

[9] G. X. Cheng and L. Shen, "A research and application of automated fully mechanized longwall mining technique for use in low seams," Journal of the China Coal Society, vol. 35, no. 9, pp. 61-62, 2009.

[10] D. W. Holman, Development of an Underground Automated Thin-Seam Mining Method, Virginia Polytechnic Institute and State University, 1999.

[11] R. Sahoo and M. M. Abdul, "Application of opto-tactile sensor in shearer machine design to recognise rock surfaces in underground coal mining," in Proceedings of the IEEE International Conference on Industrial Technology (ICIT '09), pp. 1-6, IEEE, Gippsland, Australia, February 2009.

[12] Q. G. Fan, W. Li, and C. M. Luo, "Error analysis and reduction for shearer positioning using the strapdown inertial navigation system," International Journal of Computer Science Issues, vol. 9, no. 5, pp. 49-54, 2012.

[13] N. Kursunoglu and M. Onder, "Selection of an appropriate fan for an underground coal mine using the Analytic Hierarchy Process," Tunnelling and Underground Space Technology, vol. 48, pp. 101-109, 2015.

[14] T. L. Saaty, "Decision making with the analytic hierarchy process," International Journal of Services Sciences, vol. 1, no. 1, pp. 83-98, 2008.
[15] B. Yilmaz and M. Dağdeviren, "A combined approach for equipment selection: F-PROMETHEE method and zero-one goal programming," Expert Systems with Applications, vol. 38, no. 9, pp. 11641-11650, 2011.

[16] H.-T. Nguyen, S. Z. M. Dawal, Y. Nukman, and H. Aoyama, "A hybrid approach for fuzzy multi-attribute decision making in machine tool selection with consideration of the interactions of attributes," Expert Systems with Applications, vol. 41, no. 6, pp. 3078-3090, 2014.

[17] C. Guray, N. Celebi, V. Atalay, and A. G. Pasamehmetoglu, "Oreage: a hybrid system for assisting and teaching mining method selection," Expert Systems with Applications, vol. 24, no. 3, pp. 261-271, 2003.

[18] J. P. Liu, C. S. Ji, and H. Li, "Evaluation of coal-mining methods with weighted gray clustering analysis," Journal of China Coal Society, vol. 26, no. 5, pp. 493-495, 2001.

[19] D. S. Zhang, J. X. Zhang, and X. C. Zhang, "Fuzzy evaluation on coal seam geological condition of coal face," Journal of Systems Engineering, vol. 17, no. 3, pp. 252-256, 2002.

[20] W. H. Gao and L. D. Yang, "Application of fuzzy synthetic evaluation method to geological conditions of fully mechanized mining," Systems Engineering-Theory \& Practice, no. 12, pp. 117-123, 2001.

[21] M. Ataei, H. Shahsavany, and R. Mikaeil, "Monte Carlo Analytic Hierarchy Process (MAHP) approach to selection of optimum mining method," International Journal of Mining Science and Technology, vol. 23, no. 4, pp. 573-578, 2013.

[22] H. Karimnia and H. Bagloo, "Optimum mining method selection using fuzzy analytical hierarchy process-Qapiliq salt mine, Iran," International Journal of Mining Science and Technology, vol. 25, no. 2, pp. 225-230, 2015.

[23] S. Alpay and M. Yavuz, "Underground mining method selection by decision making tools," Tunnelling and Underground Space Technology, vol. 24, no. 2, pp. 173-184, 2009.

[24] T. L. Saaty, The Analytic Hierarchy Process, McGraw-Hill, Pittsburgh, Pa, USA, 1980.

[25] J. P. Brans, P. Vincke, and B. Mareschal, "How to select and how to rank projects: the PROMETHEE method," European Journal of Operational Research, vol. 24, no. 2, pp. 228-238, 1986.

[26] I. Vukotic and V. Kecojevic, "Evaluation of rope shovel operators in surface coal mining using a Multi-Attribute DecisionMaking model," International Journal of Mining Science and Technology, vol. 24, no. 2, pp. 259-268, 2014.

[27] J. Hu and Y. Jiang, "PROMETHEE method applied in the evaluation of urban air environmental quality," Journal of University of Shanghai for Science and Technology, vol. 34, no. 4, pp. 318-322, 2012.

[28] D. DuBois and H. Prade, "Operations on fuzzy numbers," International Journal of Systems Science, vol. 9, no. 6, pp. 613626, 1978.

[29] R. U. Bilsel, G. Büyüközkan, and D. Ruan, "A fuzzy preferenceranking model for a quality evaluation of hospital web sites," International Journal of Intelligent Systems, vol. 21, no. 11, pp. 1181-1197, 2006.

[30] R. R. Yager, "A procedure for ordering fuzzy subsets of the unit interval," Information Sciences, vol. 24, no. 2, pp. 143-161, 1981.

[31] M. Goumas and V. Lygerou, "An extension of the PROMETHEE method for decision making in fuzzy environment: ranking of alternative energy exploitation projects," European Journal of Operational Research, vol. 123, no. 3, pp. 606-613, 2000.

[32] J. P. Brans and P. H. Vincke, "A preference ranking organization method," Management Science, no. 31, pp. 647-656, 2002. 


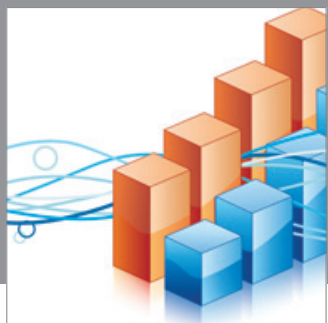

Advances in

Operations Research

mansans

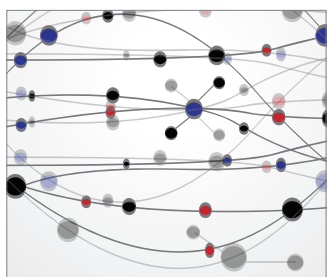

The Scientific World Journal
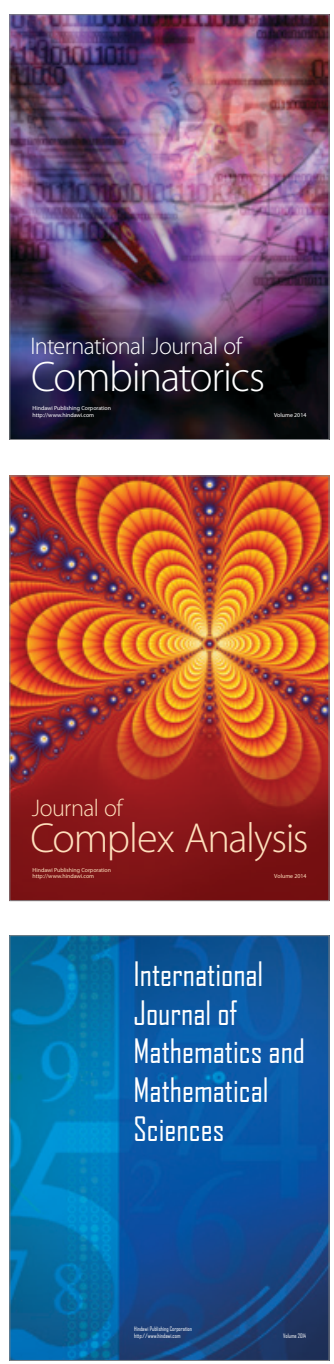
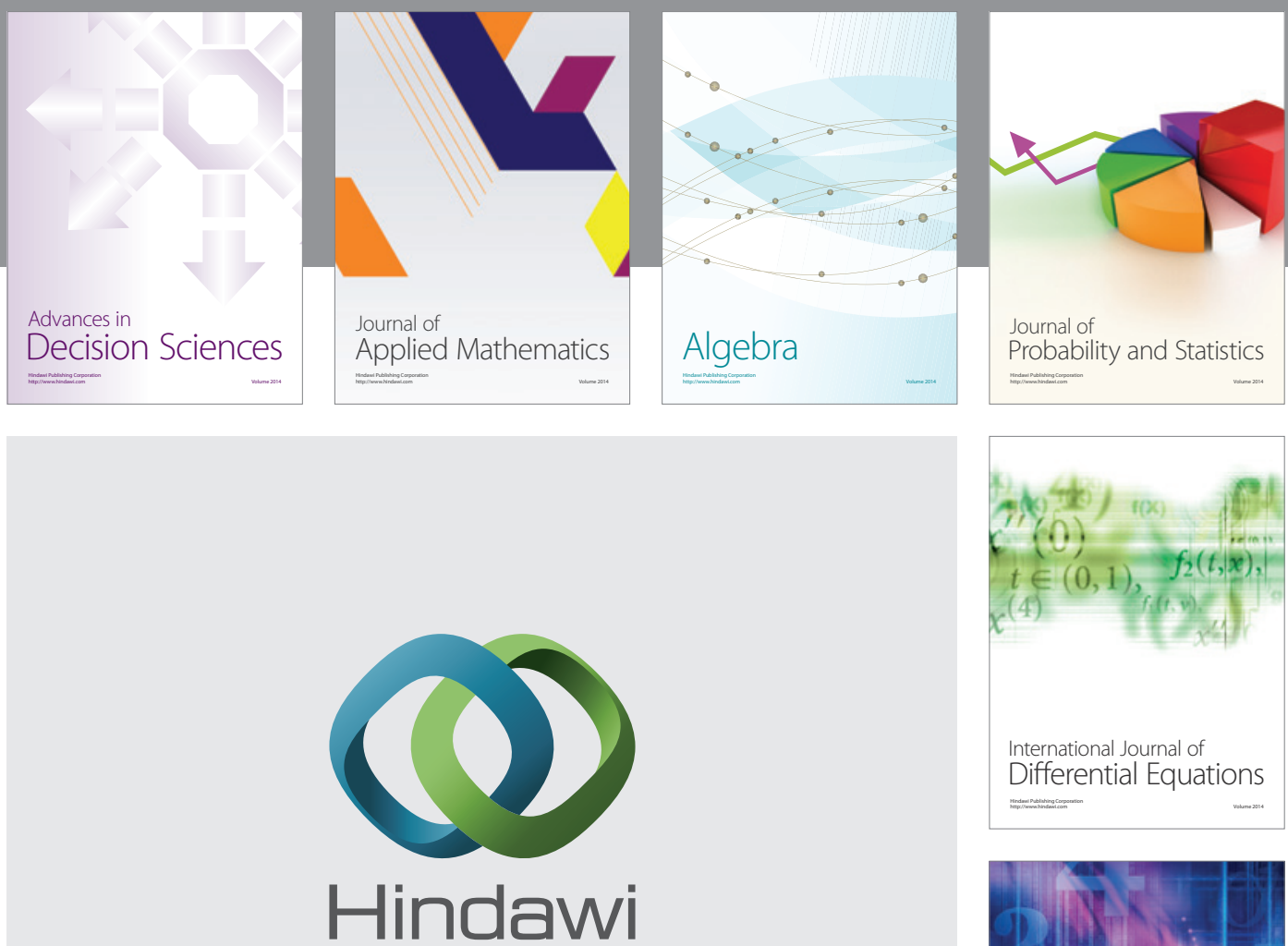

Submit your manuscripts at http://www.hindawi.com
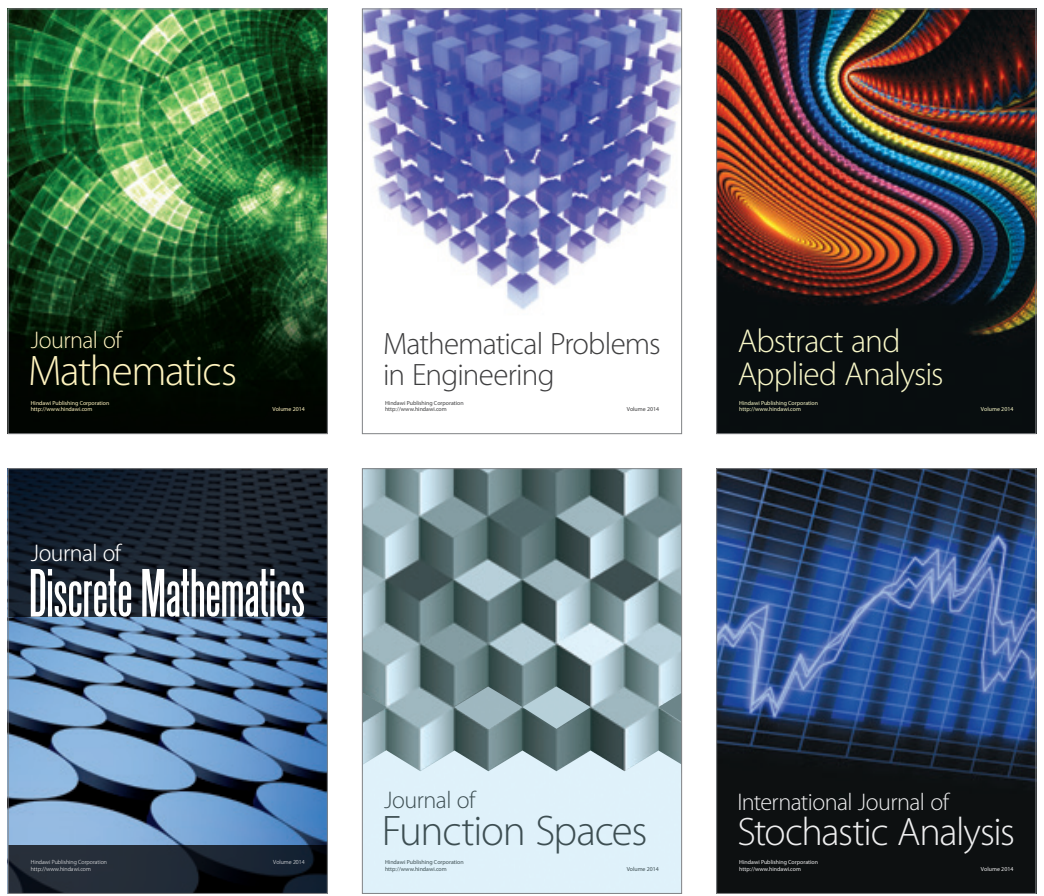

Journal of

Function Spaces

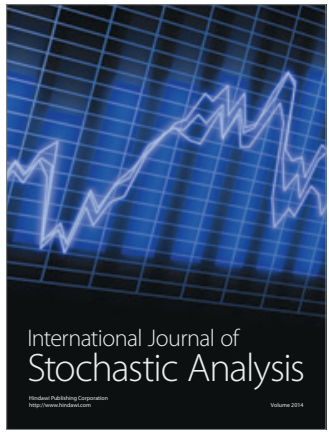

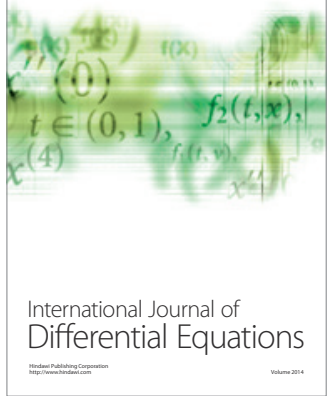
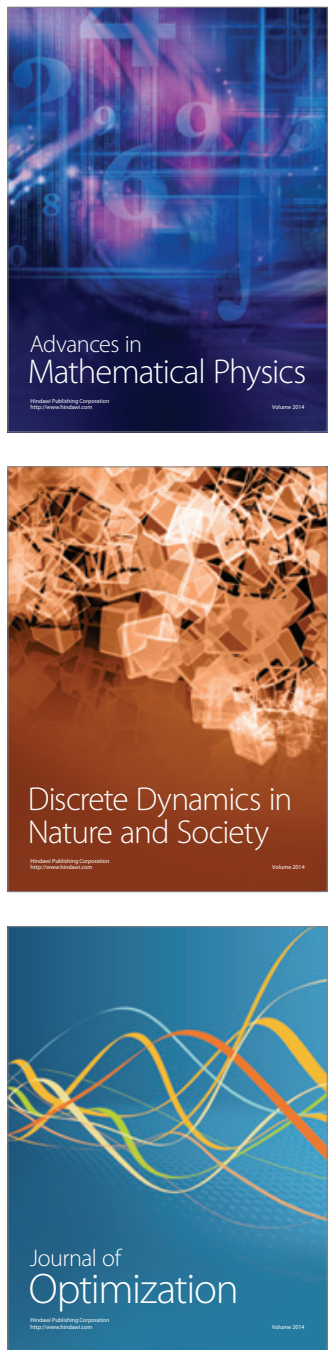